\title{
Europhysics Letters
}

When Europhysics Letters was launched, the objective was to develop an international journal of high quality that, whilst serving the world, would have a European character. The 'European ideal' was much in mind, but it was an outward looking view. The response from authors soon was exceeding by far the expectations and the Editorial Board became flooded with manuscripts. The sources? Out of curiosity we listed the countries of the authors figuring in a recent issue taken at random - that of 10 ctober. They are as follows: Romania, France (3), Switzerland (2), Spain (2), Japan, Portugal, Fed. Rep. of Germany (3), China (2), UK, Israel, USSR, Denmark, Italy. (The number in brackets indicates the number of letters where the country appears in the author list.) It would be difficult for a single issue to contain more despite the 96 pages it comprises!

The transnational appeal of the journal is evident and the physics community has applauded the quality. Moreover, the backlog of contributions that built up as a result of the dramatic success of the journal has now been absorbed, and the initial aim of having a maximum of three months between submission in acceptable form and publication, has become routine again. This has been achieved by increasing the number of pages published this year from the calculated 1600 to 2800 .

Such an explosive growth has had its repercussions, not least financially, but the decision was taken not to increase subscriptions in 1987 retrospectively, but allow our subscribers to profit from the situation. For the future, however, subscription rates will have to reflect the real value of the journal and the publishing programme will be adapted to the demand which is still increasing though at a lower pace.

From 1 January 1988, the journal will be published as a series of volumes of a standard size of 768 pages. For the next 12 months, three volumes are foreseen, but the intention is to adjust the rate to the influx of good papers. Refereeing will be as strict as in the past and the editors will be ruthless in regard to length! The subscription rate for libraries for 1988 has been fixed at Sw.Fr. 350. - per volume, i.e. Sw.Fr. 1050. - for the year, which is a stiff increase, but in terms of the price per page is still less than had originally been projected. Individual Ordinary Members of EPS, for their personal use may buy the journal in 1988 for Sw.Fr. 95. - + Sw.Fr. 30. - contribution to postage. This is for the year! A bargain indeed.

Now that the launching difficulties are behind us, all that is needed is a modest increase in circulation and everybody will gain. A little pressure from members on those central and departmental libraries that are still behind the times can make this European venture a total success. Have them place their order with 'Les Editions de Physique', Ave. du Hoggar, Z.I. de Courtaboeuf, BP 112, F-91944 Les Ulis Cedex.

\section{Hewlett-Packard Europhysics Prize 1988}

The 1988 Hewlett-Packard Europhysics Prize for outstanding achievement in solid state physics has been awarded to

J. Georg Bednorz

and K. Alex Müller

of the Zurich Research Laboratory of IBM at Rüschlikon, Switzerland for their discovery of high temperature superconductivity. The prize worth Sw.Fr. 20.000.- donated by Hewlett-4 Packard will be presented during the 8 th General Conference of the Condensed Matter Division which will be held in Budapest from 6-9 April 1988.

In view of the enormous impact their discovery has had on condensed matter physics, with new groups forming all over the world to study the type of material they introduced, it seems almost superfluous to give any explanation of their work. However, for the record, let us note that while traditional wisdom had regarded $30 \mathrm{~K}$ as the absolute maximum theoretical temperature for the onset of superconductivity, Bednorz and Müller, pursuing their systematic research on oxides, found with a layered oxide of copper, barium and lanthanum, superconductivity at $35 \mathrm{~K}$. The discovery was fundamental, a completely new field of physics was opened, and within days almost of their announcement, superconductivity at over $90 \mathrm{~K}$ had been found in similar ternary oxides containing rare earth elements.

\section{Guide to Entries}

In 1987 Europhysics News is publishing two Meetings Issues: in March and October. Urgent up-datings have also been included in the June Directory Issue in the form of advertisements charged at Sw.Fr. 100.per insertion.

The events listed here are those for which details were received at the EPS Secretariat before the end of September 1987. In general, all events interesting European physicists which take place after the beginning of November 1987 are quoted.

The listings are in date order divided into two categories - Conferences and Schools although the distinction between these two categories is not always evident. Europhysics Conferences, i.e. meetings organized by EPS, its Divisions and Sections, are printed in bold type and boxed. Events sponsored by EPS are printed in bold type; Europhysics Study Conferences have an E preceding the date.

For inclusion in the March 1988 issue, organizers should send details to the EPS Secretariat by 17 February at the latest. Even if full details are not yet available, the preliminary information should be given.

Application for sponsorship should be made to the EPS Secretariat in Geneva as early as possible.

\section{Key to Abbreviations}

A: Application deadline

Ab: Abstracts deadline

inv.: restricted to invited applicants

NP: no proceedings will be published

P: Papers deadline (information on proceedings has not been received)

PP: proceedings will be published.

The information is given in this order:

\section{Date}

Venue

Title

Contact for information

A: .... / Ab: .... / PP, NP or P: Papers deadline / Number of participants / inv. / languages other than English / Sponsorship / Conference fee.

4 after the date indicates an important amendment to the last published entry. 


\section{Conferences}

\section{7}

22-23 Oct.

Braunschweig, FRG

Symp. on Industrielle Anwendungen optischer Messtechnik

Reimund Torge, c/o Carl Zeiss, Postfach 1369 , D-7082 Oberkochen

26-28 Oct. Williamsburg, VA, USA LEOS-IEEE/OSA Tunable Solid-State Lasers Topical Meeting

Optical Society of America, 1816 Jefferson Place, N.W., Washington, DC 20036

1-5 Nov. Atlantic City, NJ, USA APS/OSA Int. Laser Science Conference

Willaim C. Stwalley, lowa Laser Facility, Univ, of lowa, lowa City, IA 52242

Ab: 12.6 .87

1-6 Nov.

Cambridge, MA, USA

Advances in Intelligent Robotics Systems

in assoc. with IECON' 87

SPIE, POB 10, Bellingham, WA 98227

PP: 5.10 .87

\section{9-12 Nov.}

Kyoto, Japan

19th Yamada Conf. on Ordering and Organization in lonic Solutions

N. Ise, Dept. of Polymer Chem., Kyoto Univ. Kyoto 606

16-20 Nov.

Hadron Structure ' 87

Smolenice, CSSR

S. Olejnik, Inst. Phys., EPRC, Slovak Acad. Sci., Dubravska cesta, CS-842 28 Bratislava

\section{6-20 Nov.}

Cannes, France

Int. Symp. on Technologies for Optoelectronics

Judith Prado, ANRT, 16 av. Bugeaud, F-75116 Paris

\section{7-11 Dec.}

New Delhi, India

7th Int. Conf, on Thin Films

P.B. Barna, Eötvös Lorand Physical Society,

P.O. Box 240, H-1368 Budapest, Hungary PP / IUVSTA, IUPAP

\section{4-18 Dec.}

Lake Buena Vista, FL, USA

12 th Int. Conf. on Infrared and Millimeter Waves

K.J. Button, Box 72, MIT, Cambridge, MA 02139-0901

15-16 Dec.

Faraday Symposium: Molecular Vibrations

Mrs. Y.A. Fish, Royal Society of Chemistry,

Burlington House, London W1V OBN

A: Nov. 87 / PP: $15.8 .87 / 100$

\section{6-18 Dec.}

Bristol, UK

Solid State Physics

Meetings Officer, The Institute of Physics,

47 Belgrave Square, London SW $1 \times 80 \times$

Reading, UK 


\section{4-18 March}

52nd Physikertagung (DPG)

Karlsruhe, FRG

Arbeitskreis Festkörperphysik (with ÖPG)

- Dünne Schichten

Difmann, Inst. f. Angew. Physik der Univ., Universitätsstr. 31 D.8400 Regensburg

Kynamik und Statistische Physik (with NNV D-7750 Konstanz

- Halbleiterphysix

U. Rössier, Inst. f. Theor. Physik der Univ., Universitätsstr. 31, D-8400 Regensburg

- Magnetismus

H. Warlimont, Vacuumschmelze GmbH, Grüner Weg 37 . - 6450 Hanau

K. Urban, Inst. f. Festkörperforschung der KFA, Postfach 1913 D-5170 Jülic

- Oberflächenphysik (with OGV/SGV)

A. Bradshaw, Fritz-Haber-Inst., Faradayweg 4-6, D-1000 Berlin 33

- Tiefe Temperaturen (with Kryotechnik)

H.F. Hoenig. Siemens AG - ZFE 1, Postfach 3240 D.8520 Erlangen

- Vakuumtechnik (with OGVISGV

G. Reich, Leybold Heraeus GmbH, Bereich VG 4

- Forschungstorderung in Europa (Beratender Ausschuss der

H. Reiner, SEL, Lorenzstrasse 10, D-7000 Stuttgart 40

Energietechnik (Physikalische Fragen) Arbeitskreis Energie der DPG

Stichel, Fak. f. Physik der Univ., Postfach 8640

D. 4800 Bielefeld 1

Ab: 1.12 .87

15-17 March Meylan (Grenoble), France

Silicon on Insulator Workshop

D. Bensahel \& G. Bomchil, CNET-CNS, BP 98 , F-38240 Meylan Cedex

A: Dec. 87 /Ab: 27.5.87/PP: Dec. 87 / 150/inv. French

16-18 March

Teilchenphysik (DPG)

Freiburg i. Br., FRG

S. Brandt, FB7 Physik der Universität,

Postfach 101240, D-5900 Siegen

Ab: 1.12 .87

16-18 March

Tokyo, Japan

Int. Symp. on Neutrino Mass and Related Topics

T. Ohshima, Inst. f. Nuclear Study, Univ. of Tokyo,

Tanashi, Tokyo 188

17-19 March

New Directions in Spectrophotometry

S.J. Adelman, Dept. of Physics, The Citadel

IAU Charleston, SC 29409

\section{0-27 March}

Novosibirsk, USSR

Physics of Wave Collapses

S.K. Turitsyn, Inst. of Automation and

Electrometry, Universitetskiy Prospect 1

SU-630090 Novosibirsk

A: 1.1.88 / NP / 40 / inv. / 40 Roubles

21-25 March

Berlin (West)

Kern- und Mittelenergiephysik (DPG with BNV, NNV, DFS, SFS

W. von Örtzen, Hahn-Meitner-Inst.

Glienicker Strasse 100, D-1000 Berlin 39

Ab: 1.12 .87

21-25 March

DPG Meeting (with OPG)

- Atomphysik

Hotop, Fachbereich Physik der Univ., Postfach 3049, D-6750 Kaiserslautern

FW. Röllgen, Inst. f. Physikalische Chemie der Univ. Wegeler Strasse 12, D-5300 Bonn 1 Wegeler Strasse

E. Tiemann, inst. A

st. f. Atom. und Molekülphysik der Univ, Appelstrasse

von der Linde, FB 7 Physik der Univ., Gesamthochschule Universitätsstr. 2, D-4300 Essen 1

Ab: 1.12 .87

21-25 March

Garching, FRG

Very Large Tel

copes and their Instrumentation -

Miss Christina Stoffer, ESO, Karl-Schwarzschild-Str. 2 $\mathrm{AU}$ D-8046 Garching

21-25 March

Karsdorf nr. Dresden, GDR

7 th Int. Lohrmann Colloquium on Geodetical Astrometry

Technische Universität Dresden, Sektion Geodäsie u Kartographie, Lohrmann-Observatorium. IAU
21-25 March New Orleans, LA, USA
General Meeting of the American Physical Society

American Physical Society, 335 East 45th St.

New York, NY 10017

21-26 March

Galway, Ireland

IUTAM Symp. on Elastic Wave Propagation

M.F. McCarthy, Dept. of Mathematical Physics,

Univ. College Galway, Galway

22-25 March

Birmingham, UK

Optics - ECOOSA '88

Meetings Officer. The Institute of Physics,

47 Belgrave Square, London SW1X $80 X$

Ab: $16.10 .87 / P P$

\section{3-25 March}

Aussois, France

Topical Meeting on Optical Bistability (OBIV)

W.J. Firth, Dept. of Physics and Appl. Phys.

Univ. of Strathclyde, 107 Rottenrow,

Glasgow G4 ONG, UK

Ab: 15.12.87 / 150 / FF 1000. - before 23.2.88, 1200. - after; 500. - students before 23.2.88 600.- after

28-29 March

Windisch, Switzerland

Spring Meeting of the Swiss Physical Society

J. Martin, EPFL, Lab de Génie Atomique

$\mathrm{CH}-1015$ Ecublens-Lausanne

Ab: 31.1.88 / P: 29.3.88/150/French, German

\section{8-30 March}

Durham, UK

Faraday Discussion No. 85: Solvation

M.C.R. Symons, Dept. of Chemistry, Univ, of Leicester, Leicester LE1 7RH

A: Feb. 88/Ab: 31.5 .87 /PP: $15.12 .87 / 200$

28-30 March Davis, CA, USA
3rd Univ. of California Conf. on Statistical Mechanics

Davis, CA, USA

C. Garrod, Physics Dept., Univ. of California

Davis, CA 95616

28-30 March

Trieste, Italy

Adriatico Conf on the Impact of Digital Microelectronics and Microprocessors on Particle Physics

international Centre for Theoretical Physics, POB 586, 1-34100 Trieste

Ab: 12.12 .87

\section{8-30 March}

anta Fe, NM, USA

Topical Meeting on Integrated \& Guided Wave Optics (IGWO '88)

Optical Society of America, 1816 Jefferson Place, N.W. Washington DC 20036

Ab: 20.11.87/PP

\section{8-31 March}

Kristallographie (DPG

J. Felsche, FB Chemie der Universität

Postfach 5560, D-7750 Konstanz

Ab: 1.12 .87

31 March - 3 Apri

43rd Annual Meeting of the Physical Society of Japan

Physical Society of Japan, Room 211, Kikai-Shinko Building, 3-5-8 Shiba-Koen, Minato-Ku, Tokyo 105 Ab: 18.12 .87

April Johannesburg, South Africa

on Beam Analysis VIII

Conference Secretariat, S, 440, POB 395

Pretoria 0001

IUPAP

4-8 April

Orlando, FL, USA

SPIE's 1988 Technical Symp. Southeast on Optics,

Electro-Optics and Sensors

SPIE, POB 10, Bellingham, WA 98227-0010

PP: 7.3 .88

5-8 April

Cambridge, UK

Acoustics ' 88

J.E. Ffowcs Williams, Engineering Dept., Univ. of Cambridge, Trumpington Street. PP Cambridge CB2 $1 \mathrm{PZ}$

6-8 April

Bristol, UK

Magnetic Resonance in Colloid and Interface Science T. Cosgrove, School of Chemistry, Univ. of Bristol Cantock's Close, Bristol BS8 1TS Ab: $1.11 .87 / \mathrm{NP}: 1.11 .87$
6-9 April
Budapest, Hungary
8th General Conf. of the Condensed
Matter Division of EPS
N. Kroo, Central Research Inst. for
Physics, POB 49, H-1525 Budapest

6-9 April

Trieste, Italy

2nd Int. Meeting on Large-Scale Structure and Motions in the Universe

F. Mardirossian, Dipartimento di Astronomia, via Tiepolo 11, 1-34131 Trieste

A: $30.3 .88 /$ PP: $15.3 .88 / 150$

11-14 April

Salford, Manchester, UK

1st European Vacuum Conference

J.S. Colligon, Dept. of Electronic and Electrical Eng. IUVSTA niv. of Salford, Salford M5 4WT

\section{2-15 April}

Tucson, AZ, USA

4th Topical Meeting on Optical Interference Coatings Optical Society of America, 1816 Jefferson Place N.W., Washington DC 20036

$A b: 31.12 .87$

18-21 April

Washington, DC, USA

Spring Meeting of the American Physical Society

American Physical Society, 335 East 45th St.

New York, NY 10017

Hannover, FRG

Optronic 88: Int. Laser-Kongress in Hannover in conjunction with Hannover fair

Optronic 88, Magazin-Verlag, Friedrichstr. 71 D.6242 Kronberg

German

24-28 April

Shoresh, Israel

8th Int. Workshop on Photon-Photon Collisions

Uri Karshon, Dept, of Nuclear Physics,

Weizman Inst. of Science, Rehovot 76100

A: $1.1 .88 / A b: 1.2 .88 / P P / 150 /$ inv

25-28 April

Bad Honnef, FRG

Workshop on Recent Advances in the Understanding of Nuclear Structure through Studies in the $\mathrm{Zr}$ Region K. Sistemich, IKP/KFA Jülich, Postfach 1913 , D-5170 Jülich

inv.

25-29 April

Anaheim, CA, USA

LEOS/IEEE/OSA Conf, on Lasers and Electro-Optics (CLEO '88)

Optical Society of America, 1816 Jefferson Place, N.W., Washington, DC 20036

26-28 April

London, UK

Fibre Optics ' 88 /6th Int. Conf, and Exhibition on Fibre Optics and Optoelectronics

Conference Office, Sira Ltd, South Hill, Chislehurst, Kent BR7 5EH

27-29 April

Lakewood (Denver), CO, USA

10th Symp. on Applied Surface Analysis

A.W. Czanderna, Solar Energy Research Inst.

1617 Cole Boulevard, Golden, CO 80401

28-30 April

Aix-les-Bains, France

9th Workshop on Grand Unification (WOGU)

J. Boratav, DPhPE//SEPh, CEN-Saclay, F-91191 Gif-sur-Yvette Cedex

2-6 May Jülich, FRG

8 th Int. Conf. on Plasma Surface Interactions in Controlled Fusion Devices

Ms M. Spittler-Wilden, KFA Jülich, POB 1913

D-5170 Jülich

5-6 May

Rio de Janeiro, Brazil

Latin-American Conf. on High Temperature Superconductivity

R. Nicolsky, Instituto de Fisica, UFRJ, CP 68528 , Rio de Janeiro 21944, Brazi

16-20 May

Riga, USSR

IUTAM Symp. on Liquid Metal Magnetohydrodynamics

J. Lielpeteris, Inst. of Physics, Latvian SSR Acad. of Sci., 229021 Riga, Salaspils

or R. Moreau, Ecole Nat. Sup. d'Hydraulique de Grenoble, B.P. 95 ,

F-39402 St. Martin-d'Hères Cedex, France 


\section{6-20 May}

Dubrovnik, Yugoslavia th Europe

M.M. Skoric, Boris Kidric Inst. of Nuclear Sciences, POB 522, YU-11001 Beograd

$A b: 22.1 .88 / P: 25.3 .88$

\section{6-20 May}

Bad Aussee, Austria

9th European Experimental NMR Conference

$\mathrm{H}$. Sterk, Karl-Franzens-Universität, Inst. f. Organische Chemie, Heinrichstrasse 28, A-8010 Graz

A: 1.4 .88 / PP / OES 1500

18-20 May

Belfort, France

3e Colloque National de Visualisation et de Traitement d'Images

Colloque Visualisation, I.U.T., B.P. 527

Rue Engel Gros, F-90016 Belfort

A: 15.2.88/French / FF 1000.-

20-23 May

Bologna, Italy

Int Symp on Modern Cosmology in Restrospect (in association with 3rd ESO-CERN symposium)

Silvio Bergia, c/o Conference Service srl, Via Tagliapietre 18/B, I-40123 Bologna

23-27 May

Kazimierz, Poland

11 th Warsaw Symp. on Elementary Particle Physics: New Theories in Physics

Z. Ajduk, Inst. of Theor. Physics, University, Hoza 69, PL - 00-681 Warszawa

23-27 May

Taipei, Taiwan, ROC Int. Conf. on Medium and High-Energy Nuclear Physic W.Y.P. Hwang, Dept. of Physics, Carnegie-Mellon Univ. Pittsburgh, PA 15213, USA

23-27 May

Gull Lake, MI, USA

5 th Gull Lake Int. Conf. on Phase Transitions in Nuclea Collisions

Laszlo P. Csernai, NSCL/Cyclotron Lab., Michigan State 120 Univ., East Lansing, MI 48824-1321

24-27 May

IUTAM Symposium Transsonicum III

Göttingen, FRG

J. Zierep, Inst. f. Strömungslehre, Univ. Karlsruhe, Kaiserstr. 12, D-7500 Karlsruhe

\section{4-27 May \\ Arcachon, France \\ 4th EPS Liquid State Conf. on the Hydro- dynamics of Dispersed Media \\ F. Carmona, Centre de Recherche Paul \\ Pascal, CNRS, Domaine Universitaire, \\ F-33405 Talence Cedex \\ $100 /$ French}

31 May - 3 June

Kiev, USSR

Int. Conf. on Physics of Transition Metals

V.S. Mikhalenkov, Inst. of Metal Physics, Acad. of Sciences of the Ukrainian SSR, Vernadsky Str. 36, sU-252 142 Kiev 142 250/ PP / Russian

May or June Williamsburg, VA, USA

1988 Linear Accelerator Conference

C.W. Leemann, CEBAF, 12070 Jefferson Ave. Newport News, VA 23606

June Montreal, Quebec, Canada Congress of the Canadian Association of Physicists Ms. M. Jento, Canadian Ass. of Physicists, 151 Slate Street, Suite 805, Ottawa, Ont. K1P $5 \mathrm{H} 3$

5-11 June Boston, MA, USA

13 th Int. Conf. on Neutrino Physics and Astrophysics (NEUTRINO 88)

Dept. of Physics, Tufts University, Medford, MA 02155 A: $1.3 .88 / \mathrm{PP}$

6-8 June

Laser Technologies in Industry

Porto, Portugal

Olivério D.D. Soares, Lab. Fisica, Fac. Ciencias, P-400 Porto

A: April 88 / PP: May 88 / 300 / French, Portuguese / ESC 20000. - IOM; 25000.- others; 9000. - students; incl. proc.

6-10 June

Tel Aviv, Israel

Landau Memorial Conf, on the Frontiers of Physics

A. Voronel, School of Physics, Tel Aviv University Tel Aviv 69978

A: 1.3.88/PP / 150 / US\$ 130.- IOM;

150. - others; 20. - students; incl. proc.
6-10 June

Infrared Technology and Applications

London, UK

Conference Office Sira Ltd, South Hill,

Chislehurst, Kent BR7 5EH

6-11 June 4

3. Conférence Internationale sur les Collisions Noyau-Noyau

C. Detraz, GANIL, BP 5027, F-14021 Caen Cedex

A: Feb. 88 / PP: March 88 / approx. FF 1200.incl. proc.

7-11 June

1st European Particle Accelerator Conference

S. Tazzari, Lab. Naz. dell'INFN,

Via E. Fermi 40, C.P. 13, 1-00044 Frascati

Ab: 16.12.87 / 400 / approx. SFR 200. - ; incl. proc.

12-17 June
19th Int. Symp. on Multiparticle Production

D. Schiff, LPTPE, Bât. 211, Université Paris Sud,

F-91405 Orsay Cedex

$\mathrm{PP} /$ inv. / FF 3000. - all incl.

13-16 June

Umeå, Sweden

11th European Conf. on Thermophysical Properties

Gunnar Bäckström, Dept. of Physics, S-901 87 Umeå

Ab: Jan. 88/SKR 1600.

13-16 June

Oslo, Norway

Annual Meeting of the Norwegian Physical Society

T. Engeland, Dept. of Physics, Univ. of Oslo, $\mathrm{POB} 1948$ Blindern, N-0316 Oslo

A: 1.5 .88 / Ab: 1.5 .88 / NP / $200 /$ Nor. / NKR 500.-

14-16 June

Oulu, Finland

3rd IFAC/IFIP/IEA/IFORS Conf. on Man-Machin Systems: Analysis, Design and Evaluation

Finnish Soc. of Automatic Control, POB 165 SF-00101 Helsink

PP: 31.12.87 / approx. SFR 600.-

15-18 June

Düsseldorf, FRG

Dynamics Days G. Eilenberger, Inst. f. Festkörperforschung
der Kernforschungsanlage Jülich GmbH Postfach 1913, D-5170 Jülich 1

A: 30.4 .88 / Ab: 30.4 .88 / NP / 200/ DM 30.

19-24 June

Gargnano, Italy

19th Europhysics Conf. on Macromolecular Physics: Polymer Crystals: Morphology, Kinetics and Applications

G.C. Alfonso, Ist. di Chimica Industriale, Univ. di Genova, Corso Europa 30 , I-15132 Genova

100 / LIT 300000. - IOM; 315000. others; 250000. - students: incl. proc.

\section{9-24 June}

Workshop on Spin Polarized Quantum System

M. Rasetti, I.S.I., Villa Gualino, Viale Settimio Severo 65 , I-10133 Torino

20-23 June

10th Symp. on Thermophysical Properties

J.V. Sengers, Inst. for Phys. Science and Techn. Univ, of Maryland, College Park, MD 20742

Ab: 1.12 .87 / PP: 23.6 .88

20-24 June Shanghai, China

BAIL V: 5 th Int. Conf. on Boundary and Interior Layers - Computational and Asymptotic Methods

Pauline McKeever, POB 5, 51 Sandycove Road,

Dun Laoghaire, Co. Dublin, Ireland

US\$260. - incl. proc., before $31.1 .88 ; 320$. - after

20-24 June

14th Int. Laser Radar Conference

Innichen-San Candido, Italy

.

I-50127 Firenze

Ab: 31.1.88 / LIT 230000. - / IAMAP

21-25 June

Topical Conf. Physics in Collision VIII

P. Strolin, Dip. Fisica and INFN, Pad. 20, Mostra d'Oltremare, 1-80125 Napoli

26 June - 2 July Santa Fe, NM, USA Int Conf. on Science and Technology of Synthetic Metals (ICSM 88)

M. Aldissi, Los Alamos Nat. Lab., POB 1663, MS K764, Los Alamos, NM 87545 PP / 600
27-30 June

Paris, France

13th Int. Sy Vaccum

A. Septier, CNAM, 292 rue Saint-Martin. F-75141 Paris Cedex 03

PP: 15.3.88 / 150 / FF 1800. - ; 1000.- students; incl. proc.

27-30 June

Canterbury, UK

th Int. Conf. on Dielectric Materials, Measurements and Applications

Institution of Electrical Engineers, Savoy Place, London WC2R OBL

PP: 15.1 .88

28-30 June

Wroclaw, Poland

9th Int. Wroclaw Symp. on Electromagnetic Compatibility

EMC Symposium, Box 2141, PL - 51-645 Wroclaw 12

\section{8-30 June}

Uranus Colloquium

Pasadena, CA, USA

J.T. Bergstrahl, JPL, Calif. Inst. of Technology, IAU

\section{4-7 July} Karlsruhe, FRG

BEAMS '88: 7th Int. Conf. on High-Power Particle Beams

G. Kessler, Kernforschungszentrum Karlsruhe POB 3640, D-7500 Karlsruhe 1

Ab: 31.12.87 / PP: April 88 / DM 500.- before 1.4.88; 580. - after; incl. proc

4-8 July Paris, France

11th Int. Conf. on Atomic Physics (ELICAP

S. Haroche, Lab. de Spectroscopie Hertzienne de I'E.N.S., 24 rue Lhomond, F-75231 Paris Cedex 05 inv.

4-9 July Aix-en-Provence, France

4th Int. Symp. on Small Particles and Inorganic Clusters (I.S.S.P.I.C. 4)

M.F. Gillet, Lab. Microscopie Diffractions, Fac. Sciences Techniques St. Jérôme, Ave. Escadrille Normandie-Niemen, F-13397 Marseille Cedex 13

A: 15.4.88/ Ab: 15.4.88/PP / 250/ FF 1000. IOM; 1200. - others; 700. - students

5-8 July Paris, France 4th IAP Astrophysics Meeting: Modelling of the Stellar Environment: How and Why?

C. Magnan, IAP, 98 bis blvd. Arago, F-75014 Paris IAU

11-14 July

Lisbon, Portugal

4th Int. Symp. on Applications of Laser Anemometry to Fluid Mechanics

D.F.G. Durao, Dept. of Mech. Eng., Inst. Superior Técnico, Avenida Rovisco Pais, P-1096 Lisboa Ab: 16.12.87

11-14 July

Prague, Czechoslovakia

11th Discussion Conf, on Macromolecules: Chemical and Physical Phenomena in the Ageing of Polymers

P. Cefelin, Inst. of Macromolecular Chemistry, Czechoslovak Acad. of Sci., Heyrovskehe namesti 2, CS-16206 Praha 6-Petriny

11-15 July

Vancouver, BC, Canada Joint INTERMAG and Magnetism and Magnetic Materials Conf.

Technical Activities Dept., IEEE, 345 East 47th Street, New York, NY 10017, USA

11-16 July

Nonlinear Processes in Vlasov Plasmas

F. Doveil, PMI, Ecole Polytechnique, F-91128 Palaiseau

A: 1.4.88 / Ab: 1.4.88/ PP: 11.6.88 / 100/French / FF 1000. - incl. proc.

\section{2-15 July}

20th EGAS Conference

Graz, Austria

L. Windholz, Inst, f. Experimentalphysik, Techn. Univ. Graz, Petersgasse 16, A-8010 Graz

A: 30.4.88/ PP: $31.5 .88 / 200 /$ OES 1400.-

\section{2-15 July}

Grenoble, France

Int. Conf, on Neutron Scattering (ICNS '88)

J. Schweizer, Centre d'Etudes Nucléaires,

DRF/SPh-MDN, 85 X, F-38041 Grenoble Cedex 
12-15 July

ICEC 12, Sales Plus, 4 Hubbard Road, Houndmills, Basingstoke RG21 2UH

Ab: 15.1 .88 / f 195. - before $31.5 .88 ; 220 .-$ after: incl. proc.

\section{7-27 July}

Swansea, UK

9th Congress of the Int. Association of Mathematical Physics

A. Truman, Dept. of Math. and Computer Science, Univ. College of Swansea, Singleton Park Swansea SA2 8PP

\section{8-21 July}

Frankfurt/Main, FRG

6th Int. Conf. on Crystal-Field Effects and Heavy-Fermion Physics

W. Assmus, Physikalisches Inst. Univ. Frankfurt, POB 11 19 32, D-6000 Frankfurt 11

$A b:$ Marh $88 /$ PP: June 88

18-22 July

Tokyo, Japan

Int. Quantum Electronics Conference (IOEC ' 88 ) OITDA, 20th Mori Building, 7-4 Nishi-Shimbashi 2-chome, Minato-ku, Tokyo 105

18-29 July Helsinki, Finland

27th COSPAR Plenary Meeting and Associated Activities

Z. Niemirowicz,

51, bd. de Montmorency, F-75016 Paris, France

A: 31.5.88/Ab: 10.1.88 / PP: 18.7.88/1200/French / US\$150.-

20-23 July Leuven, Belgium

2nd Int. Symp. on High Field Magnetism

F. Herlach, Lab v, Lage Temperaturen en Hoge-Veldenfysika, Celestijnen 200 D, B-3030 Leuven

Ab: 1.3.88 / PP: 21.7.88 / FB 5000.- IOM; 5500. - others; incl. proc.

24-29 July Lausanne, Switzerland

European Conf. on Computers in Education

B. Levrat, Centre Universitaire d'Informatique, 12 rue du Lac, $\mathrm{CH}-1207$ Genève

Ab: 30.10.87/PP: 15.5.88/French, Russian, Spanish / IFIP

25-29 July

Int. Conf. on Magnetism (ICM '88)

Int. Conf. on Magnetism (ICM '88) Univ. Paris Sud, F-91405 Orsay Cedex

A: 1.6.88 / Ab: 31.1.88/FF 1500.- before 1.5.88 1800. - after; incl. proc.; 800. - students

25-29 July

Kyoto, Japan

5 th Int. Conf. on Clustering Aspects in Nuclear and Subnuclear Systems (Cluster ' 88 )

Y. Abe, Research Inst. for Fundamental Physics, PP Kyoto Univ., Kyoto 606

\section{5-29 July}

9th Int. Conf. on Spectral Line Shapes (a) Copernicus Univ., ul. Grudziadzka 5/7, PL - 87-100 Torun

27-29 July

Bloomington, IN, USA

IAU Colloquium No. 106: Evolution of Peculiar Red Giant Stars

H.R. Johnson, Astronomy Dept., Swain West 319 Indiana University, Bloomington, IN 47405

27-30 July

Williamstown, MA, USA

IAU Colloquium No. 105: The Teaching of Astronomy

J.R. Percy, Dept. of Astronomy Univ. of Toronto, Toronto, Ont. M5S 1A1, Canada

August Novosibirsk, USSR

8th Int. Conf. on Synchrotron Radiation Utilization (SR 88)

A.N. Skrinsky, USSR Academy of Sciences, Siberian Division, Inst. of Nuclear Physics, SU-Novosibirsk 90

1-4 Aug

Nuclear Methods in Magnetism (Satellite to ICM '88)

G.M. Kalvius, Physik E 15, TU München, D-8046 Garching

A: March 88 / PP: 1.8.88 / 300 / DM 260.- IOM 300. - others; incl. proc.

1-5 Aug.

Burgundy, France

12th Int. Colloquium on Magnetic Films and Surfaces

1.B. Puchalska, Lab. de Magnétisme et d'Optique des Solides CNRS, F-92195 Meudon Principal Cedex
2-11 Aug.

Baltimore, MD, USA

30th General Assembly of Int. Astronomical Union

Jean-Pierre Swings, IAU-UAI Secretariat,

61 Ave. de I'Observatoire, F-75014 Paris

A: $15.5 .88 /$ US\$ 150. - before $15.5 .88 ; 200$. - after

4-10 Aug.

24th Int. Conf. on High Energy Physics

Munich, FRG

Föhringer Ring 6, D-8000 München 40

PP / 1200 / inv. / IUPAP

8-12 Aug.

Perth, Australia

5th Marcel Grossmann Meeting: Recent Devel. in Theor. and Exptl. General Relativity, Gravitation and Relativistic Field Theories

David Blair, Physics Dept., Univ. of Western Australia, Perth 6009 IUPAP

8-12 Aug.

5th Trieste Semiconductor Symposium, and

4 th Int. Conf. on Superlattices, Microstructures and Microdevices

G. Döhler, Univ. of Erlangen, Erwin-Rommel-Str. 1, D-8520 Erlangen

(Satellite to 19th Int. Conf. on Physics of

Semiconductors, 15-19.8 in Warsaw

8-12 Aug.

Würzburg, FRG

Application of High Magnetic Fields in Semiconducto Physics

(Satellite to 19th Conf. 15-19.8 in Warsaw)

G. Landwehr, Phys. Inst. der Univ.

Röntgenring 8, D-8700 Würzburg

10-12 Aug.

Linköping, Sweden

Shallow Impurities in Semiconductors

B. Monemar, Condensed Matter Physics,

Linköping Univ., S-581 83 Linköping

15-19 Aug.

Warsaw, Poland

19th Int. Conf. on the Physics of Semiconductors

J. Kossut, Inst. of Physics. Polish Acad. of Sciences,

Al. Lotnikow 32/46, PL - 02-668 Warszawa

A: 30.5.88 / Ab: 15.3.88/ 1000 / IUPAP /

US\$190. - incl. proc.

15-19 Aug.

Sarajevo, Yugoslavia

14th Summer School and Int. Symp on the Physics of lonized Gases - SPIG ' 88

L. Tanovic, Electrical Eng. Faculty, Univ. of Sarajevo, POB 327, YU-71000 Sarajevo

15-19 Aug.

Palo Alto, CA, USA

IAU Colloquium No. 104: Solar and Stellar Flares

B.M. Haisch, Div. 91-20, Bldg 255, Lockheed Palo Alto Res. Lab., 3251 Hanover Str., Palo Alto, CA 94304

15-19 Aug. Dominion Observatory, Victoria, Canada IAU Colloquium No. 107: Algols

M.J. Plavec, Dept. of Astronomy, Univ. of California, Los Angeles, CA 90024

18-23 Aug. Non-prics Workshop on Fror

A. Patkos, Dept. of Atomic Phys., Eötvös Univ. Puskin u. 5-7, H-1088 Budapest

18-25 Aug.

Lille, France

Meeting of the Radiation Commission of the Int. Association of Meteorology and Atmospheric Physics (IAMAP)

J.E. Harries, Rutherford Appleton Lab., Chilton, Didcot, Oxon. OX11 0QX, UK

21-27 Aug.

Grenoble, France

17 th Int. Congress of Theoretical and Applied Mechanics (ICTAM 88)

Denis Caillerie, Inst. de Mécanique de Grenoble, Domaine Universitaire, BP No. 68 . F-38402 St. Martin-d'Hères

$\mathrm{Ab}: \mathbf{8 . 2 . 8 8}$ / PP / FF 1500.-

22-23 Aug.

Warsaw, Poland

Int Conf, on High Pressure in Semiconductor Physics L. Konczewicz, Zaklad Wysokich Cisnien PAN

ul. Sokolowska 29/37, PL - 01-142 Warszawa

(Satellite meeting to 19th Conf. 15-19.8 in Warsaw)

22-25 Aug.

Nonlinear Optical Properties of Materials

Optical Society of America, 1816 Jefferson Place, N.W., Washington, DC 20036

22-25 Aug.

Cambridge, MA, USA

High Resolution Spectroscopy of Cosmic Plasmas

Paul Gorenstein, Harvard-Smithsonian Center $f$

Astrophysics, 60 Garden Street, Mail Stop 4.

IAU
22-26 Aug

Zurich, Switzerland

CIRP 4: 4th Int. Conf. on Infrared Physics

F.K. Kneubühl, Infrared Physics Lab., Inst. of Quantum Electronics, ETH, Hoenggerberg, $\mathrm{CH}-8093$ Zürich

A: $1.1 .88 / \mathrm{Ab}: 1.1 .88 / \mathrm{PP}: 15.5 .88$ /SFR 340.before $1.6 .88 ; 380$. - at meeting; incl. proc.

22-26 Aug.

Vienna, Austria

7th Int. Symp. on Gas Flow and Chemical Lasers

ARGELAS, Mostgasse 3, A-1040 Wien

22-26 Aug.

Berlin, GDR

Optical Nonlinearities and Bistability in

Semiconductors

R. Enderlein, Berlin, DDR

(Satellite to 19th Conf. 15-19.8 in Warsaw)

22-26 Aug.

Budapest, Hungary

15th int. Conf. on Defects in Semiconductors

G. Ferenczi, Budapest

(Satellite to 19th Conf. 15-19.8 in Warsaw)

22-27 Aug.

Budapest, Hungary

12 th Int. Conf. COLING ' 88

COLING 88 Secretariat, c/o MTESZ Congress Bureau, Kossuth tér 6-8, H-1055 Budapest

Ab: 10.12 .87 / PP: 30.4 .88

24 Aug. - 3 Sept.

Varenna, Italy

Joint Varenna-Abastumani Workshop on Plasma Astrophysics

E. Sindoni, Int. School of Plasma Physics, 16 Via Celoria, 1-20133 Milano

A: $15.6 .88 /$ PP / $100 /$ US $\$ 250$. - incl. proc

28 Aug. - 2 Sept.

28 Aug. - 2 Sept. Vienna, Austria Stristallographie $u$ A-1060 Wien

29 Aug. - 1 Sept.

Zurich, Switzerland

1st European Conf. on Applications of Polar Dielectrics and Int. Symp. on Applications of Ferroelectrics

H. Arend, Lab. of Solid State Physics, ETH, $\mathrm{CH}-8093$ Zürich

29 Aug. - 2 Sept.

11th Int. Mass Spectrometry Conference

Ecole Polytechnique, F-91128 Palaisea

29 Aug. - 2 Sept.

Parma, Italy

Int. Conf. on Lattice Defects in Insulating Crystals

Rosanna Capelletti, Dipto. di Fisica, 300 Via M. d'Azeglio 85, 1-43100 Parma

29 Aug. - 2 Sept. Cavtat-Dubrovnik, Yugoslavia Symp. on the Electronic Structure and Properties of Molecules and Molecular Crystals

Zvonimir B. MaksiC, The Rudjer Boskovic Inst. POB 1016, YU-41001 Zagreb

29 Aug. - 2 Sept. Prague, Czechoslovakia

10th IUPAC Conf. on Chemical Thermodynamics IUPAC Conference, POB 119, CS-160 O0 Praha 6

29 Aug. - 3 Sept.

Ghent, Belgium

8th Int. Conf. on Positron Annihilation ICPA-8

Lab. for Nuclear Physics, Rijksuniversiteit Gent, Proeftuinstraat 86, B-9000 Gent

A: 15.5 .88 / Ab: 15.5 .88 / US $\$ 60 .-10 M$ 70. - others; 60 . - students

29 Aug. - 4 Sept. Bucharest, Romania 3rd Europhysics Conf. "Trends in Quantum Electronics" (TQE '88)

I.A. Dorobantu, I.N. Mihailescu, POB MG-54, R-76900 Magurele, Bucharest $V$

A: $15.7 .88 /$ Ab: $15.6 .88 /$ PP: $4.9 .88 / 600 /$ US $\$ 70$. IOM; 85. - mbrs. $4 \mathrm{~b}$ ); 110 . - others; incl. proc.

30 Aug. - 2 Sept. Toulon, France

Topical Meeting on Optical Computing

G. Lebreton, ARAVI, Univ. de Toulon et du Var, Ave. de I'Université, F-83130 La Garde Ab: $28.2 .88 / \mathrm{PP}: 2.9 .88 / \mathrm{ICO}$

30 Aug. - 2 Sept. Brioni, Yugoslavia Classical Dynamics in Atomic and Molecular Physics P. Grujic, Inst. of Physics, POB 57, YU-11001 Beograd,

or Ms. Milica Djordjevic, Inst. of Physics, Maksima Gorkog 118, YU-11080 Zemun

A: 1.11 .87 / PP / 80 / US \$ 150. - IOM; 170.- others: 50. - students; incl. proc. 
30 Aug. - 3 Sept.

Cavalese (Trento), Italy

8th Italian Conf. on General Relativity Gravitational Physics

S. Zerbini, Dept. of Physics of the Univ.

1.38050 Povo (Trento)

Sept.

Bologna, Italy

Int. Interdisciplinary Meeting on Chemistry and Physics of Electrified Interfaces

Istituto Chimico "G. Ciamician", Università degli Studi, Via Selmi 2, 1-40126 Bologna 80

3-6 Sept.

Mragowo, Poland

High Pressure Chemical Synthesis and Physical Transformations

J. Jurczak, Inst. of Organic Chemistry, Polish Academy of Sciences, Kasprzaka 44, PL - 01-224 Warszawa

A: May 88/Ab: April 88 / NP / 150 / US\$ 325.-

200. - students; incl. board, lodg.

\section{5-8 Sept. \\ Bologna, Italy \\ ECOSS-10: European Conf. on Surface \\ Science \\ O. Bisi, Dipto. di Fisica, Univ. Modena \\ Via Campi 213/A, I-41100 Modena \\ A: 1.6.88 / Ab: 15.3.88/ PP: 15.6.88/500}

5-9 Sept.

London, UK

11th Int. Conf. on Raman Spectroscopy (ICORS '88)

John F. Gibson, Royal Soc. of Chemistry,

Burlington House, London W1V OBN

A: 18.7 .88 / PP: 18.3 .88 / IUPAC

5-9 Sept.

Balatonfüred, Hungary

Diffusion in Metals and Alloys (DIMETA-88)

F.J. Kedves, Roland Eötvös Physical Society,

POB 433, H-1371 Budapest

A: $15.4 .88 /$ Ab: $15.4 .88 / P P: 5.9 .88 / 250 /$ US $\$ 160$.

5-9 Sept.

Assisi, Italy

MOLEC VII - Dynamics of Low Energy Molecular Collisions

Vincenzo Aquilanti, Dipto. di Chimica dell'Università, 1-06100 Perugia

A: $15.5 .88 / \mathrm{Ab}: 15.5 .88 /$ US\$ 400 . - all incl

5-9 Sept.

Torun, Poland

7th Polish Spectroanalytical Conf., and

10th Conf, on Analytical Atomic Spectroscopy

J. Fijalkowski, Inst. of Nucl. Chemistry and Technology, Polish

Dorodna 16, PL - 03-195 Warszawa

5-10 Sept.

Bechyne Castle, CSSR

European Symp. on Few-Body Physics: Mesons and

Light Nuclei IV

R. Mach, Nuclear Physics Inst., Czech. Acad. Sci.

CS-25068 Rez

A: $1.4 .88 /$ PP: $5.9 .88 / 110 /$ approx. US\$120. - all incl.

7-9 Sept.

Minneapolis, MN, USA

Cosmic Abundances of Matter

C.J. Waddington, Physics Dept., Univ. of Minnesota

116 Church St. S.E., Minneapolis, MN 55455 IAU

12-15 Sept.

Hannover, FRG

EQEC '88: European Conf. on Quantum Electronics

Inst. of Quantum Optics, Univ. of Hannover.

Welfengarten 1, D.3000 Hannover 1

Ab: 1.5 .88

12-16 Sept.

Munich, FRG

Int. Conf. on "Teaching Solid State Physics"

K. Luchner, Sektion Physik, Univ. München IUPAP

Schellingstrasse 4, D-8000 München 40

12-16 Sept.

Riva del Garda, Italy

6 th Int. Conf. on Surface Modification of Metals by Ion Beams

SM2IB Conference Secretariat, I.R.S.T.

PP 1-39050 Povo (Trento)

\section{2-16 Sept.}

Aussois, France

6th Workshop on Interactions of Molecular Beams and Surfaces

Jean Lapujoulade, CEN Saclay, D.Ph.G.SPAS

F-91191 Gif-sur-Yvette Cedex

NP / 100

\section{3-15 Sept.}

Exeter, UK

Spectrom Temperature

A.C. Legon, Dept. of Chemistry, Univ. of Exeter, Exeter EX4 4OD

A: Aug. 88/PP: 15.5.88/200
13-16 Sept.

Structure and Reactivity of Surfaces

C. Morterra, Ist. di Chimica Fisica,

Corso Massimo d'Azeglio 48, I-10125 Torino $\mathrm{PP} / 250$

16-19 Sept.

London, UK

Int. Conf. on Physical Interpretations of Relativity Theory

M.C. Duffy, Mech. Eng. Dept., Sunderland Polytechnic, Chester Road, Sunderland, SR1 3SD

Ab: Jan. 88 / PP: May 88/120/French, German approx. $€ 120$. - all incl.

19-23 Sept.

Utrecht, NL

15th Symp on Fusion Technology (15th SOFT)

Mrs. A. Nijsen-Vis, FOM-Inst. v. Plasmafysica

"Rijnhuizen", POB 1207, NL-3430 BE Nieuwegein Ab: 15.4 .88 / PP: 19.9 .88

\section{9-23 Sept. \\ Hamburg, FRG}

Int. Conf. on Optical Science and Engineering

Europtica Services I.C., 16 ave. Bugeaud, F-75116 Paris

Ab: 8.2.88/ PP: 22.8.88/1500 Europtica, SPIE

19-25 Sept.

Leipzig, GDR

4th Conf. on Radioisotope Application and Radiation Processing in Industry

Central Inst. of Isotope and Radiation Research of the Acad. of Sci. of the GDR, Permoserstr. 15, DDR-7050 Leipzig

US\$ 150.- ; 90.- students; incl. proc.

25-30 Sept. Lausanne, Switzerland

20th Europhysics Conf. on Macromolecular Physics

and 3rd Lausanne Polymer Meeting: Physical Mechanisms in Polymer Failure

H.H. Kausch, Chaire de Polymères EPFL, Ch. de Bellerive 32, CH-1007 Lausanne

A: 30.6.88/Ab: 28.2.88 / NP /

SFR 300. - IOM; 320, - members 4b) 350. - others; 250. - students

26-29 Sept. Cape Cod, MA, USA

4th Topical Meeting on Short Wavelength Coherent Radiation: Generation and Applications Optical Society of America, 1816 Jefferson Place, N.W., Washington DC 20036 $A b: 3.6 .88$

3-7 Oct.

4th Int. Symp. on Radiation Physics

Mrs. M.K. Sarcinella, Rue Augusta

$2516-2^{\circ}$ and - cj 22, 01412 Sao Paulo

Ab: 15.5 .88 / PP / US\$ 200. - before 15.8.88;

220. - after; incl. proc.

3-8 Oct.

Wroclaw, Poland

9 th Int. Symp. on Exoelectron Emission and Applications

Jan Lesz, Inst. of Experimental Physics, Univ. of Wroclaw, Cybulskiego 36, PL - 50-205 Wroclaw

PP / US\$ 300. - incl. board, lodg.

9-14 Oct.

Chicago, IL, USA

Symp. on Luminescence Science and Technology

Electrochemical Society, 10 South Main Street. Pennington, NJ 08534

PP: 1.5 .88

10-14 Oct.

Bad Honnef, FRG

Int. Workshop on Nuclear Dynamics at Medium and High Energies

Ulrich Mosel, Inst. f. Theor. Physik, Univ. Giessen, D-6300 Giessen

12-19 Oct.

12th Int. Conf. on Plasma Physics and Controlled Nuclear Fusion Research

Conf. Service Section, IAEA, POB 100, A-1400 Wien, Austria

16-21 Oct. Houston, TX, USA

IMEKO XI: 11th Triennial World Congress of the International Measurement Confederation Instrumentation for the 21st Century

IMEKO Secretariat, POB 457

$\mathrm{H}-1371$ Budapest, Hungary

PP: 31.7 .87

26-30 Oct.

Cannes, France

4th Int. Colloquium on Welding and Melting by Electron and Laser Beams

CEA, DTECH-STA, F-91191 Gif-sur-Yvette Cedex

30 Oct. -4 Nov.

Hollywood, FL, USA

Meeting of the APS Div, of Plasma Physics

W.W. Havens Jr, APS, 335 E. 45th St. New York, NY 10017

31 Oct. - 4 Nov.

Santa Clara, CA, USA

OPTICS '88: OSA Annual Meeting

Optical Society of America, 1816 Jefferson Place, N.W. Washington, DC 20036

\section{1-27 Nov.}

Workshop on Physics for Development

Mohammed Bilal, Dept. of Physics, Univ. of Dar-Es-Salam, Tanzania

or E. Lillethun, Dept. of Physics, Univ, of Bergen, $\mathrm{N}-5007$ Bergen, Norway

PP / 45 / inv. / French

12-14 Dec.

Bad Honnef, FRG

Orientation and Polarisation Effects in Reactive Collisions

Mrs. Y.A. Fish, Royal Society of Chemistry Burlington House, London W1V OBN

A: 1.11 .88 /Ab: 20.11 .87 / PP: $15.8 .88 / 60$

20-22 Dec.

Nottingham, UK

Annual Solid State Physics Conf.

Meetings Officer, The Institute of Physics,

47 Belgrave Square, London SW1X 80X

\section{9}

19-23 Jan.

Dallas, TX, USA

LEOS-IEE-OSA Conf on Optical Fiber Communication Optical Society of America, 1816 Jefferson Place N.W. Washington, DC 20036

13-17 February

5th Int. Wire Chamber Conference

.

Regler, Inst. f. Hochenergiephysik
Nikolsdorfergasse 18, A-1050 Wie

A: 2.1.89 / Ab: 30.11.88 / PP: Feb. 89 / 280

SFR 230,-;85, - students; incl, proc.

Mid-Feb.

Warsaw, Poland

5th Symp, on Optical Fibres and their Applications

R.S. Romaniuk, Technical Univ, of Warsaw, IPE, Nowowiejska 15/19, PL - 00-665 Warszawa

A: Dec. 88 / Ab: Dec. 88 / PP / 500 / Germ., Pol., Russ. / Free to $10 \mathrm{M}$

6-9 March

Nice, France

9 th General Conf. of the Condensed Matter Division of EPS

J.P. Laheurte, Lab. de la Matière Condensée, Univ. de Nice, F-06034 Nice cedex

13-17 March

Venice, Italy

16th EPS Conf. on Controlled Fusion and Plasma Physics

M. Fontanesi, Ist. di Fisica del Plasma, CNR,

Via Bassini 15, 1-20133 Milano

20-24 March

St. Louis, MO, USA

March Meeting of the American Physical Society

W.W. Havens Jr., APS, 335 E. 45th St. New York, NY 10017

11-13 April

Liverpool, UK

Catalysis by Well Characterised Materials

R.W. Joyner, Leverhulm Centre f. Innovative Catalysis, Univ of Liverpool, POB 147. Liverpool L69 3BX

A: March 89 / Ab: 31.5.88 / PP: 15.12.88/ 200

24-28 April

Baltimore, MD, USA

IQEC '89: LEOS Conference

Optical Society of American, 1816 Jefferson Place, N.W., Washington, DC 20036

24-28 April

CLEO '89: LEOS-IEEE-OSA Conf. on Lasers and Electro-Optics

Optical Society of America, 1816 Jefferson Place, N.W., Washington, DC 20036

24-28 April

Bamberg, FRG

Comets in the Post-Halley Era

R. Newburn, JPL, "64-667”, Oak Grove Drive IAU Pasadena, CA 91109, USA

24-28 April

Paris, France 
1-4 May

Baltimore, MD, USA

Spring Meeting of the American Physical Society

W.W. Havens Jr., APS, 335 E. 45th Str. New York, NY 10017

15-20 May

Montreal, Canada

Int. Symp. on Weak and Electromagnetic Interactions in Nuclei (WEIN '89)

P. Depommier, Univ. of Montreal, POB 6128, Station A, Montreal, Quebec, Canada $\mathrm{H} 3 \mathrm{C} 3 \mathrm{~J} 7$

12-15 June

Helsinki, Finland

5 th Int. Congress on Tribology - EUROTRIB 89

Kenneth Holmberg, Technical Res. Centre, VTT/KOT,

Metallimiehenkuja 6, SF-02150 Espoo

A: $1.5 .89 / \mathrm{Ab}: 1.7 .88 / \mathrm{P}: 1.4 .89$

10-15 July

Plasma Phenomena in Solar Physics

M.A. Dubois, DRFC-STGI, CEN Cadarache,

F-13108 St. Paul-lez-durance Cedex

A: 1.4.89/ Ab: 1.4.89/ PP: 10.6.89 / 50 / FF 500.incl. proc.

26 July - 1 Aug.

New York City, NY, USA

16 th Int. Conf. on the Physics of Electronic and Atomic Collisions (ICPEAC)

W.R. Newell, Dept. of Physics, Univ. College of London, Gower Street, London WC1E 6BT, UK

August

Prague, Czechoslovakia

8th Int. Conf, on Hyperfine Interactions

M. Finger, Fac. of Mathematics and Physics, 400

Charles Univ., Ke Karlovu 3, CS-121 16 Praha 2

6-13 Aug.

Stanford, CA, USA

1989 Int Symp on Lepton-Photon Interactions

R.E. Taylor, SLAC, POB 4349, Stanford, CA 94305

IUPAP

13-18 Aug.

Rio de Janeiro, Brazi

GR-12: 12th Conf. of the Int. Society of General Relativity and Gravitation

M. Soares, Centro Brasileiro de Pesquisas Fisicas, Rua Xavier Sigaud 150, CEP 22920 Rio de Janeiro - RJ

14-18 Aug.

Honolulu, HI, USA

9 th Int. Conf. on Vacuum Ultraviolet Radiation Physics (VUV9)

Charles S. Fadley, Dept. of Chemistry, Univ. of Hawaii,

2545 The Mall, Honolulu, HI 96822

20-26 Aug.

Int. Conf. on High Energy Accelerators

S. Ozaki, KEK, Oho-machi, Tsukuba-gun,

Ibaraki-ken 305

IUPAP

28 Aug. - 1 Sept.

Saarbrücken, FRG

7 th Int. Meeting on Ferroelectricity - IMF-7

H. Müser Fachbereich 11 - Physik der Univ. des

Saarlandes, D-66 Saarbrücken

24-29 Oct.

Antibes, France

CASIA 89 - European Conf. on Applications of

Surface and Interface Analysis

Mme J. Fauvet, Société Française du Vide,

19 rue du Renard, F-75004 Paris

$\mathrm{PP} / 300$

\section{0}

\section{4-8 Sept. Amsterdam, NL Bth General Conf. of the European Physical Society \\ Mrs. Louise Roos, FOM-Inst. for Atomic and Molecular Physics, POB 41883 NL-1009 DB Amsterdam}

\section{4-26 Sept.}

St. Helier, Jersey, UK

European Gallium Arsenide Conference

Meetings Officer, The Institute of Physics,

47 Belgrave Square, London SW1X 8QX

Oct. (tentative)

Singapore

25 th Int. Conf. on High Energy Physics

K.K. Phua, Physics Dept., National Univ. of Singapore, Kent Ridge, Singapore 0511

\section{Schools}

8-19 Feb.

Trieste, Italy

Workshop on Functional-Analytic Methods in Complex Analysis and Applications to Partial Differential Equations

Int. Centre for Theoretical Physics, POB 586,

1.34100 Trieste

15 Feb. - 18 March

Trieste, Italy

Workshop on Applied Nuclear Theory and Nuclea Model Calculations for Nuclear Technology Applications

Int. Centre for Theoretical Physics, POB 586. I-34100 Trieste

\section{7}

2-20 Nov.

College on Soil Physics

international Centre for Theoretical Physics, POB 586, 1.34100 Trieste A: 30.4 .87

9 Nov. - 18 Dec.

Trieste, Italy

College on Riemann Surfaces

Iternational Centre for Theoretical Physics,

POB 586, 1-34100 Trieste

23 Nov, - 18 Dec.

2nd College on Cloud Physics and Climate

International Centre for Theoretical Physics,

POB 586, 1-34100 Trieste

7-9 Dec.

Bad Honnef, FRG

Forschungsmanagement in der Physik XII

J. Debrus, Physikzentrum Bad Honnef,

Hauptstrasse 5, D-5340 Bad Honnef

A: 16.11 .87 / 60 / Germ. / approx. DM 140.

28 Dec. - 6 Jan.

Jerusalem, Israel

5th Winter School for Theor. Phys.: Statistical

Mechanics of Membranes and Surfaces

Tsvi Piran, Inst. of Advanced Studies, Hebrew Univ., Jerusalem 91904

US\$ 400. - incl. board, lodg

\section{8}

11 Jan. - 5 Feb.

College on Variational Problems in Analysis
Int. Centre for Theoretical Physics, POB 586 1-34100 Trieste

13-27 Jan.

Karpacz, Poland

24th Winter School of Theoretical Physics: Stochastic Methods in Mathematical Physics

W. Karwowski, Inst. of Theoretical Physics, Wroclaw Univ., Cybulskiego 36, PL - 50-205 Wroclaw A: 30.11 .87 / PP / 100

18 Jan. - 12 Feb.

Trieste, Italy

2nd School on Advanced Techniques in Computational Physics

Int. Centre for Theoretical Physics, POB 586, -34100 Trieste

6-13 Feb.

Vanezza di Bondone, Italy

1st Winter Course of ISODARCO (Int. School on Disarmement and Research on Conflicts)

Carlo Schaerf, Dip. di Fisica, I Università di Roma, Piazzale Aldo Moro 2, 1-00185 Roma

A: 10.1 .88 / NP / LIT 350000 -

8-13 Feb.

Folgaria, Italy

Winter European Course on Amorphous Silicon: Fundamental Properties and Applications

F. Velli, 2 Via Verbano, I-21027 Ispra

A: 31.12.87 / Ab: 15.1.88 / PP / 100 / inv. /

LIT 350000.- before 30.11.87, 450000.- after;

+ LIT 300000.- hotel acc. and board.
22 Feb. - 2 March

Schladming, Austria

27th Internationale Universitätswochen f Kernphysik: Particle- and Astrophysics - Current Viewpoints

F. Widder, Inst. Theoretical Physics, Universitätsplatz 5, A-8010 Graz

A: $5.2 .88 / \mathrm{PP} / 150$

22 Feb. - 11 March

Trieste, Italy

Winter College on Laser Physics: Semiconductor Lasers and Integrated Optics

Int. Centre for Theoretical Physics, РОВ 586 , I-34100 Trieste

6-12 March Chateau Lake Louise, Alberta, Canada Lake Louise Winter Institute: Quantum Chromodynamics: Theory and Experiment

Secretary, Lake Louise Winter Institute, Dept. of Physics, Univ. of Alberta, Edmonton, Alberta T6G 2J1

A: 15.11.87 / CAN \$ 150.- incl. preceedings

14-25 March

Trieste, Italy

2nd Workshop on Optical Fibre Communication

Int. Centre for Theoretical Physics, POB 586, 1-34100 Trieste

20-26 March

Saas-Fee, Switzerland

$30^{\circ}$ Cours de Perfectionnement de l'Association Vaudoise des Chercheurs en Physique: Unités de Mesure et Constantes Physiques

J.F. Loude, Inst. de Physique Nucléaire

Univ, de Lausanne, BSP, $\mathrm{CH}-1015$ Lausanne

29 March - 7 April

Les Houches, France

Simple Molecular Systems at Very High Density

A. Polian, Physique des Milieux Condensés,

Univ. P. et M. Curie, T 13 E 4, 4 Place Jussieu,

F-75252 Paris Cedex 05

A: 31.12.87 / PP: 15.3.88 / FF 3500, - all incl.

5-13 April

Spring School: Heavy Flavour Physics

M.P. Locher, Swiss Institute for Nuclear Research,

$\mathrm{CH}-5234$ Villigen

$\mathrm{PP} / 100 /$ inv.

16-30 April

23rd Zakopane School on Physics

Zakopane, Poland

Part 1: 16-23.4: Selected Topics in Nuclear Structure R. Broda, Inst. of Nuclear Physics, PL-31 342 Krakow Part 2: 23-30.4: Condensed Matter Studies by Nuclear Methods

K. Krolas, Inst. of Physics, Jagellonian Univ.

PL-31 059 Krakow

20-30 April

Erice, Italy

Int School of Cosmic Ray Astrophysics, 6th Course: Cosmic Gamma Rays and Cosmic Neutrinos

John P. Wefel, Dept. of Physics and Astronomy,

Louisiana State Univ., Baton Rouge,

LA 70803, USA

A: 1.3 .88 / PP: a.s.a.p. / 70 / inv. / NATO / US\$ 500.all incl.

25 April - 17 June

Trieste, Italy

11 th Int. Course on the Theory of Condensed Matter

International Centre for Theoretical Physics,

POB 586, 1-34100 Trieste

A: 15.5 .87

16-20 May

Taipei, Taiwan, ROC

Spring School on Medium and High-Energy Nuclear Physics

W.Y.P. Hwang, Dept. of Physics, Carnegie-Mellon University, Pittsburgh, PA 15213, USA

23 May - 17 June

Trieste, Italy

Course on Physical Climatology and Meteorology for Environmental Application

Int. Centre for Theoretical Physics, POB 586,

1-34100 Trieste

A: 15.1 .88 
29 May - 7 June

Int. School of Crystallography, 14th Course: Crystallography of Molecular Biology

L. Riva di Sanseverino, Piazza Porta San Donato 1 1-40126 Bologna

A : 30.11.87/SFR 1400. - incl. board, lodg.

30 May - 24 June

Les Houches, France

Les Houches Summer School Session XLVIII Liquids at Interfaces

J. Zinn-Justin, Ecole de Physique Théorique, Côte des Chavants, F-74310 Les Houches

A : 1.3.88 / 50 / French / NATO / FF 3550. - all incl.

31 May - 10 June

Zakopane, Poland

28th Cracow School of Theoretical Physics

Inst. of Physics, Jagellonian Univ., Reymonta 4,

PL - 30-059 Krakow

A: $31.3 .88 / 100 /$ US\$200.-

20-24 June

Roskilde, Denmark

1st Topsoe Summerschool: Superconductivity

Jorgen Kjems, Physics Dept., Risö Nat. Lab., DK-4000 Roskilde

A: $1.5 .88 /$ Ab: $1.5 .88 /$ PP: $1.6 .88 / 40 /$

approx. DKR 3000. - all incl.

20-25 June

Wroclaw, Poland

Int. School on Excited States of Transition Elements

W. Strek, Inst. of Low Temperature and Structure

Research, PAS, POB 937, PL - 50-950 Wroclaw

$A b: 31.1 .88$ / US\$ 250. - incl. board, lodg.

20 June - 8 July

Lerici, Italy

Int. School of Physics "Enrico Fermi" 1st Course: Current Trends in the Physics of Materials

Mario Tosi, ICTP, POB 586, I-34100 Trieste

A: April $88 / \mathrm{PP} / 50$

28 June - 8 July

Varenna, Italy

Int. School of Physics "Enrico Fermi" 2nd Course: The Chemical Physics of Atomic and Molecular Clusters

G. Scoles, Dept. of Chemistry, Univ. of Waterloo, Waterloo, Ont. N2L 3GL, Canada

A: May $88 / \mathrm{PP} / 50$

28 June - 5 Aug.

Les Houches, France

Les Houches Summer School Session XLIX Fields, Strings, Critical Phenomena

J. Zinn-Justin, Ecole de Physique Théorique, Côte des Chavants, F-74310 Les Houches

A: 1.3.88 / 50 / French / NATO / FF 5100. - all incl.

1-15 July

Erice, Italy

Int. School of Materials Science and Technology, 3rd Course: Solid State Batteries of Low Dimensionality

J.R. Akridge, Eveready Battery Co., Inc., 25225 Detroit Rd., POB 45035, Westlake, $\mathrm{OH} 44145$, USA

A: 1.7 .88 / PP: $1.7 .88 / 60 /$ US\$ 450 . - all incl.

3-15 July Erice, Italy

Int. School of Materials Science and Technology, 16th Course: Silicon Materials Science and Technology

G. Harbeke, S.I.N., c/o Labs. RCA, Badenerstr. 569, $\mathrm{CH}-4048$ Zürich, Switzerland

A: $1.5 .88 / 70$

EPS Divisions, Sections and Group

Astronomy and Astrophysics Division

Solar Physics Section

Atomic and Molecular Physics Division

Atomic Spectroscopy Section

Chemical Physics

Electronic and Atomic Collisions

Molecular Physics

Computational Physics Group

Condensed Matter Division

Liquids Section

Low Temperature Physics Section

Macromolecular Physics

Magnetism

Metal Physics

Semiconductors and Insulators

Surfaces and Interfaces

High Energy \& Particle Physics Division

Interdiv. Group on Exptl. Phys. Control Systems

Interdiv. Group on Physics for Development

Nuclear Physics Division

Optics Division

Plasma Physics Division

Quantum Electronics Division
4-8 July

lon Chemistry

Departamento de Quimica,

C-XIV, Universidad Autonoma de

Madrid, Cantoblanco,

E-28049 Madrid

A: 1.6.88 / NP / 300 / PTAS 11000. - IOM, mbrs 4b); 13000. - others; 9500. - students

6-12 July

Venice, Italy

Int. School of Intermediate Energy Nuclear Physics, 6th Course

Carlo Schaerf, Dipto. di Fisica, Univ. di Roma Piazzale Aldo Moro 2, 1-00185 Roma

A: $25.5 .88 / \mathrm{PP} /$ LIT 700000 .

12-22 July

Varenna, Italy

int. School of Physics "Enrico Fermi" 3rd Course: Photoemission and Absorption Spectroscopy of Solids and Interfaces with Synchrotron Radiation R. Rosei, Dpto. di Fisica, Via Valerio 2, 1-34127 Trieste A: May 88 / PP / 50

14-25 July St. Croix, Virgin Islands, USA Advanced Study Inst. on Techniques and Concepts of High Energy Physics

T. Ferbel, Dept. of Physics and Astronomy,

Univ. of Rochester, Rochester, NY 14627

A: $15.3 .88 /$ NATO

18-28 July

Venice, Italy

ISODARCO (Int. School on Disarmament and Research on Conflicts): 12th Course

C. Schaerf, Dipto. di Fisica, I Università di Roma, Piazzale Aldo Moro 2, 1-00185 Roma A: 10.6.88/PP/LIT 700000.-

26 July - 5 Aug.

Varenna, Italy

Int. School of Physics "Enrico Fermi" 4th Course: Nonlinear Topic in Ocean Physics

A.R. Osborne, Ist. di Cosmo-Geofisica del C.N.R., Corso Fiume 4, I-10133 Torino

A: May $88 / \mathrm{PP} / 50$

8-21 Aug.

Dronten, the Netherlands

International Summer School on New Aspects in Nuclear Dynamics

J.H. Koch, Nuclear Phys. Div., the Netherlands' Physical Society (NNV), Postbus 5451,

NL-1007 Amsterdam

9 Aug. - 3 Sept. Les Houches, France Les Houches Summer School Session XL Geophysical Tomography

J. Zinn-Justin, Ecole de Physique Théorique, Côte des Chavants, F-74310 Les Houches

A: 1.3.88 / 50 / French / NATO / FF 3550. - all incl.

14-26 Aug.

Edinburgh, UK

Optical Computing

F.A.P. Tooley, Physics Dept., Heriot-Watt Univ., Riccarton, Edinburgh EH14 4AS

A: 15.5.88/PP / 100 / NATO / \& 350.- incl. board, lodg

14-26 Aug.

Banff, Alb., Canada

Canadian Ass. of Physicists' Summer Institute on Particles and Fields

A.N. Kamal, Dept. of Physics, Univ. of Alberta, Edmonton, Canada T6G 2.J
16 Aug. - 9 Sept.

Summer School on Dynamical Systems

Int. Centre for Theoretical Physics, POB 586, 1-34100 Trieste

A: 31.1 .88

29 Aug. - 8 Sept. Bechyne Castle, Czechoslovakia Int. Summer School on Hyperfine Interactions

M. Finger, Fac. of Mathematics and Physics, Charles University, Ke Karlovu 3, CS-121 16 Praha 2

\section{September}

Varna, Bulgaria

5 th Int. School on Condensed Matter Physics: Disordered Systems and New Materials

N. Kirov, Inst. of Solid State Physics,

72 Boulv, Lenin, BG-1784 Sofia

A: $15.5 .88 / \mathrm{Ab}: 15.588 / \mathrm{PP}: 1.10 .88 /$ Russian

5-9 Sept.

Puidoux, Switzerland

2nd Graduate Summer Course on Computational Physics: Numerical Methods for Parallel Vector Computers

R. Gruber, CRPP, EPFL, 21 av. des Bains, CH-1007 Lausanne

A: 1.7.87/ PP / $100 /$ SFR 300. - IOM; 330. - others

\section{6-16 Sept.}

Varenna, Italy

Int. School of Plasma Physics "Piero Caldirola": Course and Workshop on Inertial Confinement Fusion

E. Sindoni, Int. School of Plasma Physics,

16 via Celoria, I-20133 Milano

A: $15.6 .88 / \mathrm{Ab}: 15.6 .88 / 100 /$ US $\$ 300$. incl. proc.

10-17 Sept.

Sulejow, Poland

ECP '88: International School on Electrochemistry of Conductive Polymers

J. Plocharski, Inst. of Inorg. Technology,

Warsaw University of Technology

ul Noakowskiego 3, PL - 00-664 Warszawa

18 Sept, - 1 Oct.

1988 CERN School of Physic

Ms. S.M. Tracy, DG Division, CERN,

CH-1211 Genève 23, Switzerland

A: $30.4 .88 /$ PP / 100 / inv. / approx. SFR 1250. - all incl.

\section{Schools 1989}

16-25 July

Erice, Italy

Int. School of Nuclear Physics, 12th Course: The Nature of Baryons and Nuclei studied by Electron Scattering

Amand Faessler, Univ. of Tübingen,

Inst. f. Theoretische Physik,

Auf der Morgenstelle 14, D-7400 Tübingen A: 1.3.89/60/SFR 900. - incl. board, lodg.

19-28 Sept.

Skalsky Dvur, Czechoslovakia

8th Summer School on Computing Techniques ManMachine Interface in Scientific Environment

J. Nadrchal, Inst, of Physics, Na Slovance 2 CS-180 40 Praha 8

SFR 490. - IOM; 520.- others; all incl.
Europhysics News is the official journal of the European Physical Society which comprises 29 National Societies, Academies and Group, about 4000 Individual Members and 75 Associate Members. Governing bodies of EPS are the General Meeting, Council and an elected Executive Committee responsible for detailed policy. EPS promotes the collaboration of physicists throughout Europe, organising and harmonising conferences and publications, improving physics education, encouraging physics applications, awarding scholarships to sponsored schools in Erice. EPS publishes in addition to Europhys. News, Europhysics Letters (in partnership with national societies), European Journal
of Physics (in partnership with The UK Inst. of Phys.), of Physics (in partnership with The UK Inst. of Phys.), and European Conference Abstracts. Individual Members receive Europhys. News free of charge (price to insts: Sw.Fr. 90.-/a), Europhys. Lett. at Sw.Fr. 70.-/a (insts. 595.-), rebates on many other publications and on conference fees. Annual EPS membership fee for Individual Members belonging to an EPS member society is: Sw.Fr. 44.-; independent members: Sw.Fr. 132.-; members of a Collaborating Society: Sw.Fr. 55.-

\section{Editor: E.N. Shaw}

Editorial Board:

A. Baratoff, F. James, M. Lehmann,

M. Mayor, J. Muller, M. Siegrist

Editorial and Advertising Office at the EPS Secretariat

Address: EUROPEAN PHYSICAL SOCIETY

P.0. Box 69,

CH-1213 Petit-Lancy 2

Switzerland

Telephone: Geneva (22) 931130

Telex: 428024 eps ch

Cables: europhys genève

Printed by: Pfirter frères sa

CH-1213 Petit-Lancy/Switzerland 